\title{
Cecal inoculum peritonitis: an alternative model for sepsis vascular dysfunction study.
}

\author{
Shinichi Asano ${ }^{1,2}$, Nandini D.P.K. Manne ${ }^{1,2,4}$, Geeta Nandyala ${ }^{1}$, Bing Ma ${ }^{1,2}$, \\ Vellaisamy Selvaraj ${ }^{1,2}$, Arvapalli Ravikumar ${ }^{1}$, Kevin M. Rice $^{1}$, and Eric Blough ${ }^{1,2,3}$ \\ ${ }^{1}$ Center for Diagnostic Nanosystems, Marshall University, Huntington, WV, USA \\ ${ }^{2}$ Department of Pharmacology, Pharmaceutical Sciences and Research, School \\ of Pharmacy, Marshall University, Huntington, WV, USA \\ ${ }^{3}$ Department of Pharmacology, Physiology and Toxicology, Marshall University, \\ Joan C. Edwards School of Medicine, Marshall University, Huntington, WV, \\ ${ }^{4}$ Department of Public Health, Marshall University, Marshall University, \\ Huntington, WV, USA
}

Key words: sepsis, vascular inflammation, $\mathrm{K}^{+}$channel, and nitric oxide

Corresponding author:

Current address:

Shinichi Asano, Ph. D.

Center for Cardiovascular and Respiratory Sciences

School of Medicine, West Virginia University

1 Medical Center Drive

P.O. Box 9227

Morgantown, WV 26506

Phone: 304-293-8878

Fax: 304-293-7105

Email: sasano@hsc.wvu.edu 


\section{Abstract}

Sepsis is a life threatening condition that is characterized by the loss of vascular reactivity. The factor(s) responsible for the diminished vascular function seen in sepsis are not well understood and are likely to differ depending upon the type septic insult. The purpose of this study was to characterize the vascular dysfunction from the rat cecal inoculum $(\mathrm{Cl})$ sepsis model using cecal ligation and puncture (CLP), and lipopolysaccharide (LPS) sepsis as reference models. Phenylephrine (PE)-induced aortic contraction was decreased in each model $(p<$ 0.05) and not normalized by L-NAME or indomethacin. However, the vascular response elicited in the $\mathrm{Cl}$ model for acetylcholine (Ach) was more similar to that seen in the CLP than the LPS model. The removal of the endothelial layer increased sensitivity to L-NAME $(p<0.05)$ in aortae from $\mathrm{Cl}$ group. Inhibition of the large conductance $\mathrm{Ca}^{2+} /$ voltage sensitive $\mathrm{K}^{+}\left(\mathrm{BK}_{\mathrm{Ca}}\right)$ channel did not normalize PE hyporesponsiveness but did abolish sepsis-induced contractile oscillation. Inhibition of the voltage dependent $\mathrm{Kv}_{1.5}$ channel was not able to reverse the vascular hyporesponsiveness, however, inhibition of the ATP dependent (K $\left.\mathrm{K}_{\mathrm{ATP}}\right)$ channel inhibition partially restored the contractile response $(p<0.05)$. Elevation of VCAM expression and aortic structural alternation were observed in each model. These results suggest that the $\mathrm{Cl}$ model may be an additional tool that could be used to investigate the mechanisms of vascular hyporesponsiveness in sepsis. 


\section{Introduction}

Sepsis is a complicated, potentially life threatening public health concern that affects over 750,000 Americans per year [1]. The underlying etiology of sepsis is incompletely understood, however it is thought that vascular dysfunction characterized by hypotension plays a key role in mediating patient survivability [2-4]. The two most commonly used models to investigate sepsis in the laboratory are the endotoxemia model using lipopolysaccharides (LPS) and the polymicrobial sepsis cecal ligation and puncture (CLP). The LPS model seems to be a favorable model for some types of vascular related studies given its severe inflammatory characteristics; however, it is unclear how well this procedure mimics the human condition [5]. The CLP model, unlike the LPS platform, uses a polymicrobial insult that is more applicable to clinical sepsis [6] but it appears to suffer from intra- and inter-laboratory variability [7]. Recent studies using the newer and less characterized cecal inoculum $(\mathrm{Cl})$ polymicrobial sepsis model have suggested that this model may produce a septic response that is more suitable than the CLP procedure in certain experiments [8-10]. Whether the mechanism(s) responsible for producing the hypotension observed in the $\mathrm{Cl}$ model $[9,11]$ are similar to that which mediate the loss of vascular reactivity seen in other the septic models has to our knowledge not been investigated.

Recent studies have identified several factors that might contribute to the vascular dysfunction seen with sepsis including alterations in nitric oxide (NO) production [12-14], increased vascular inflammation [15-17], endothelial dysfunction [18], and the dysregulation of potassium ion channel function [19-21]. 
Whether $\mathrm{Cl}$-induced peritonitis is characterized by 1) elevated vascular NO production, 2) vascular hyporesponsiveness, or 3) alterations in ion channel regulation has yet to be documented. The purpose, therefore, of this study was to characterize the $\mathrm{Cl}$ induced vascular dysfunction and if applicable, compare and contrast the degree of the vascular hyporesponsiveness produced by the different preclinical sepsis models. Our data suggest that the $\mathrm{Cl}$ model could be an additional polymicrobial study model for the investigation of sepsis vascular dysfunction. 


\section{Materials and Methods}

\section{Animal}

Ten week old male Sprague Dawley rats were purchased from Hilltop Laboratories (Scottdale, PA) and housed for 2 weeks to allow acclimation before the initiation of any experiments. Animals were housed two to a cage with 12-12 dark-light cycles at $25{ }^{\circ} \mathrm{C}$. Food and water were provided ad libitum. All animal care and experimental procedures were performed after approval by the Marshall University Institutional Animal Care and Use Committee and in accordance with the Guide for the Care and Use of Laboratory Animals [22]

\section{Sepsis/endotoxemia procedures}

Cecal inoculum (Cl) model

Animals were anesthetized using isoflurane and fecal peritonitis was induced by making a small abdominal incision followed by cecal inoculum and closure of the incision as described previously [9]. The cecal material was prepared by mixing cecal contents obtained from fresh donor rats with $5 \%$ dextrose water to yield a concentration of $600 \mathrm{mg}$ cecal material in $5 \mathrm{ml} / \mathrm{kg}$. Sham control animals underwent an identical surgery to induce fecal peritonitis, but received sterile dextrose water ( $5 \mathrm{ml} / \mathrm{kg}$, i.p.) only.

Cecal Ligation puncture (CLP) model

Similarly, animals were anesthetized using isoflurane and fecal peritonitis induced by CLP as described previously [6]. Briefly, a small incision was made to 
expose the cecum and fecal material was carefully pushed to the end of the cecum to maintain the same amount of fecal material. Thereafter, the cecum was ligated $4 \mathrm{~cm}$ from the end of the cecum with a $3 / 0$ silk ligature and two punctures were made on the cecum with a $22 \mathrm{G}$ needle. After the puncture, the cecum was placed back to the peritoneal cavity and the abdominal incision was closed using suture. Animals in the sham group underwent a laparotomy and cecal manipulation but no ligation or puncture.

\section{LPS model}

Endotoxemia was induced by i.p. injection of LPS (Sigma, St. Louis, MO, Lipopolysaccharides from Escherichia coli 055:B5) $40 \mathrm{mg} / \mathrm{kg}$ in $5 \mathrm{ml} / \mathrm{kg}$ of sterile saline $(0.9 \% \mathrm{NaCl})$ to conscious animals. Control animals received vehicle $(0.9 \%$ $\mathrm{NaCl})$ i.p. injection.

\section{Isometric tension recording}

Animals were sacrificed at $18 \mathrm{~h}$ for CLP and Cl models or at $24 \mathrm{~h}$ for the LPS model. These time points were chosen on the basis of pilot studies examining the mortality rate exhibited by the different sepsis models and corresponded to the earliest time of death observed in each of the different models. Aortic contractile function was measured as previously described with slight modifications $[23,24]$. Briefly, the aorta was removed and carefully cleaned of perivascular fat in ice cold HEPES buffered physiological saline solution containing (mM) $135 \mathrm{NaCl}, 5 \mathrm{KCl}, 2 \mathrm{CaCl}_{2}, 1 \mathrm{MgCl}_{2}, 10$ glucose, $10 \mathrm{HEPES}$ and 
5 Tris; $\mathrm{pH}$ 7.4. Two $\sim 3 \mathrm{~mm}$ wide lengths of the mid-thoracic aorta were used for contractile measurements while the rest of aortae was flash frozen in liquid $\mathrm{N}_{2}$ and saved for biochemical analysis. Aortic rings were mounted on 2 stainless pins in glass organ baths containing bicarbonate buffered physiological saline solution (PSS) containing (mM) $130 \mathrm{NaCl}, 4.7 \mathrm{KCl}, 1.18 \mathrm{KH}_{2} \mathrm{PO}_{4}, 1.17 \mathrm{MgSO}_{4}$, 14.9 $\mathrm{NaHCO}_{3}, 5.5$ Glucose, 0.026 EDTA, and $1.6 \mathrm{CaCl}_{2}$. The bath was oxygenated with $95 \% \mathrm{O}_{2}$ plus $5 \% \mathrm{CO}_{2}$ to maintain $\mathrm{pH} 7.4$ and temperature was maintained at $37{ }^{\circ} \mathrm{C}$. The contents of the baths were changed every $\sim 30 \mathrm{~min}$ through the experiments and 3-5 washes were performed between the dose response experiments. Isometric tension was continuously recorded using a force displacement transducer (FTD3, Grass Technologies, Warwick, RI) to a Grass polygraph and the data were digitized at $100 \mathrm{~Hz}$ using a Polyview $16 \mathrm{~A} / \mathrm{D}$ converter (Grass Technologies, Warwick, RI).

Aortic rings were stretched in a stepwise manner to reach an optimal tension (1.4-1.6g:separately determined by $80 \mathrm{mM} \mathrm{KCl}$ response in $200 \mathrm{mg}$ passive tension each step). After a $2 \mathrm{~h}$ equilibration period, vessel viability was determined by iso potassium PSS (KPSS: $80 \mathrm{mM} \mathrm{KCl}$ equimolar $\mathrm{NaCl}$ replacement of PSS). Endothelial function was confirmed by acetylcholine (Ach: $\left.10^{-6} \mathrm{M}\right)$ after phenylephrine $\left(\mathrm{PE}: 10^{-6} \mathrm{M}\right)$ induced contraction and Ach $\left(10^{-6} \mathrm{M}\right)$ response was calculated as a percentage of PE. The vascular contraction response was assessed by measuring the tension developed in response to the cumulative addition of phenylephrine (PE: $10^{-9}$ to $10^{-5} \mathrm{M}$ ) in the absence / presence of NG-nitro-L-arginine methyl ester (L-NAME: $\left.10^{-4} \mathrm{M}\right)$ and 
indomethacin (Indo: $10^{-5} \mathrm{M}$ ). In experiments using denuded aortic rings, the endothelium was removed by gentle rubbing using a small cotton-tip. The loss of endothelial cells was confirmed by the lack of responsiveness to Ach after precontracting the aorta with PE. The area under the curve was calculated from before and after the addition of L-NAME from each ring and was expressed as arbiter units.

In the separate experiments for the $\mathrm{K}^{+}$channel assessment, aortic rings were adjusted to optimal length in a stepwise manner and vessel viability was confirmed by KPSS. After several washes, $\mathrm{K}^{+}$channel function was assessed in aorta constricted with PE $\left(10^{-6} \mathrm{M}\right)$ in the absence or presence of different $\mathrm{K}^{+}$ channel antagonists.

\section{Immunoblot analysis}

Semiquantitiative immunodetection was performed as detailed previously [25]. Briefly, aortae were pulverized and then homogenized in RIPA buffer supplemented with $1 \mathrm{mM}$ DTT, protease and phosphatase inhibitors (Sigma, St. Louis, MO). Lysates were sonicated on ice and centrifuged at $10,000 \mathrm{~g}$ at $4{ }^{\circ} \mathrm{C}$ for $10 \mathrm{~min}$. The supernatant was removed and protein concentration was determined by 660 protein assay reagent (Sigma, St. Louis, MO). Samples were diluted in Laemmli sample buffer and equal amounts of protein were separated by SDS-PAGE, followed by electroblotting onto nitrocellulose membranes. Membranes were blocked with $5 \%$ milk in TBST for $1 \mathrm{~h}$ at room temperature, washed 3 times in TBST before incubation overnight at $4{ }^{\circ} \mathrm{C}$ with primary 
antibodies (VCAM and $\beta$-actin: Cell Signaling Technology, Beverly, MA). Membranes were then incubated with secondary antibody conjugated with HRP for $1 \mathrm{~h}$ at room temperature. An enhanced ECL was used to detect immunoreactivity using a FluorChem E System (Cell Biosciences, Santa Clara, $\mathrm{CA})$.

\section{Statistics}

Data are presented as the mean and standard error of the mean as described in the text and figure legends. Statistical comparisons were made using unpaired-or paired- $t$ tests, one- or two- way ANOVA with Bonferroni post hoc tests as appropriate. A $p<0.05$ was considered as significant. 


\section{Results}

The degree of aortic hyporesponsiveness to PE differs between sepsis models.

The mortality rates for the LPS $(n=18 / 38)$, CLP $(n=16 / 24)$ and $\operatorname{Cl}(n=$ $30 / 33$ ) were $47 \%, 67 \%$ and $91 \%$ at 6 days, respectively (Table 1). These mortality data were from several cohort studies and some of data presented in other studies were combined as current overall mortality data from septic animal models in our lab. To confirm the presence of bacteremia, blood samples were obtained at 3- and $18 \mathrm{~h}$ from the $600 \mathrm{Cl}$ animals serially diluted and cultured on blood agar plates using standard methods [26]. Relative to control animals, bacteria levels in the $\mathrm{Cl}$ animals were $\sim 18,500 \%$ and $\sim 22,500 \%$ higher at 3 - and 18- $\mathrm{h}$ respectively (data not shown). Optimal resting tension was determined in the aortae by the contraction response from $\mathrm{KCl}$-induced active tension. Increasing passive tension significantly augmented $\mathrm{KCl}$ active tension and the response plateaued between 1.4-1.6 g (Figures $1 \mathrm{~A}, \mathrm{~B}$ ). A basal tension load of 1.4-1.6 $\mathrm{g}$ was used for all subsequent experiments.

Sepsis and endotoxemia each caused hyporesponsiveness to PE $(P<$ 0.05 , Figures $2 \mathrm{~A}-\mathrm{C}$ ). The loss of tension development (Sham vs. Cl: $-57 \%$ Figure 3 A; Sham vs. CLP: $-47 \%$ Figure 3 B) was similar in the $\mathrm{Cl}$ and CLP models while the loss of aortic tension following PE stimulation appeared greater in the LPS model (Control vs. LPS $-72 \%$, Figure $3 \mathrm{C}$ ). To assess the potential contribution of NO to the vascular hyporeactivity, vessels were pre-treated with LNAME for 30 min $(100 \mu \mathrm{M})$ and the PE dose response was recorded. In all three 
models, L-NAME treatment augmented PE responsiveness (Figures $2 \mathrm{~A}-\mathrm{C}$ ). LNAME treatment caused a leftward shift in the half maximal effective concentration $\left(\mathrm{EC}_{50}\right)$ of $\mathrm{PE},\left(\mathrm{pEC} \mathrm{C}_{50} ; 7.13\right.$ to $7.37,6.98$ to $7.24,6.85$ to 7.33 , in the sham $\mathrm{Cl}$, sham CLP, control LPS vessels, respectively, $P<0.05$, Table 1 ). However, PE sensitivity against the corresponding control after L-NAME treatment was not changed in the septic and endotoxemia groups. In the $\mathrm{Cl}$ and CLP aortae, the Ach $(1 \mu \mathrm{M})$ response was not significantly different from the sham control group (Sham vs Cl: $72 \pm 5$ vs. $72 \pm 6 \%$, n.s. Figure $3 A$; Sham vs. CLP: $62 \pm 5$ vs. $75 \pm 10 \%$, n.s. Figure 3B) while the LPS aorta showed significantly decreased Ach response (Control vs. LPS $81 \pm 4$ vs. $54 \pm 4 \% P<$ 0.05 , Figure $3 \mathrm{C}$ ). The vascular contraction in response to PE was not affected by COX inhibition in any of the models (Figures $2 \mathrm{~A}-\mathrm{C}$ ).

\section{Sepsis vascular smooth muscle dysfunction}

It is thought that the contractile dysfunction observed at the later stages of the sepsis produced by the CLP model is caused by impairment of the vascular smooth muscle function [27]. Conversely, in the LPS model, the impairment of aortic contraction does not seem to be of smooth muscle origin [28]. To determine the effect of the $\mathrm{Cl}$ model on vascular smooth muscle cell function aortae were denuded in an effort to remove endothelial contribution. Similar to what was predicted to occur in the CLP model, we found that aortic contractile function was diminished in the denuded $\mathrm{Cl}$ aorta $(\mathrm{E}-; 1.01 \pm 0.11 \mathrm{~g} / \mathrm{mm}$ vs. $0.66 \pm$ $0.04 \mathrm{~g} / \mathrm{mm}: P<0.05$, Figure 4B). As anticipated, denudation abolished L-NAME 
sensitivity in sham control aorta. Interestingly, denuded aorta from the $\mathrm{Cl}$ animals showed a significantly increased response to L-NAME, suggesting that NO production may not be confined solely to the endothelial cells $(-0.07 \pm 0.06 \mathrm{AU}$ vs $0.45 \pm 0.03 \mathrm{AU}, P<0.05$ vs Sham E-, Figure $4 \mathrm{~B})$. Taken together, these data suggest that $\mathrm{Cl}$-induced vascular dysfunction is not only endothelial driven, but also driven by smooth muscle NOS dysregulation.

\section{Hyper activated $\mathrm{K}^{+}$channels in sepsis}

Previous data has suggested that $\mathrm{K}^{+}$channel dysfunction may play a role in the hyporesponsiveness seen in the aorta obtained from LPS treated rats [29, 30]. Similarly, the CLP sepsis model also appears to be associated with alterations in $\mathrm{K}^{+}$channel activity and calcium handling that can give rise to the development of contractile oscillations [31, 32]. This suggests the contribution of

altered the large conductance $\mathrm{Ca}^{2+} /$ voltage sensitive $\mathrm{K}^{+}\left(\mathrm{BK}_{\mathrm{Ca}}\right)$ channel activity in CLP aorta however, it is not known whether $\mathrm{BK}_{\mathrm{Ca}}$ channel activity is also dysregulated in the $\mathrm{Cl}$ model. To investigate the potential mechanisms responsible for this behavior, we first confirmed the presence of rhythmic contraction with PE $(10 \mu \mathrm{M})$, exchanged the incubation buffer, and then induced PE contractions in the presence of the $\mathrm{BK}_{\mathrm{Ca}}$ channel inhibitor penitrem $\mathrm{A}$ (300 $\mathrm{nM}$ ). Consistent with the possibility of $\mathrm{BK}_{\mathrm{Ca}}$ involvement, $\mathrm{BK}_{\mathrm{Ca}}$ channel inhibition was found to completely block the oscillatory contractile behavior observed following PE stimulation (Figure 5 B). Confirming this, we also observed a similar 
inhibition following the direct addition of penitrem A to a muscle bath containing aortae exhibiting oscillatory activity (data not shown).

Hyper-activated voltage dependent potassium (Kv) channels have been shown to alter membrane potential and basal tone in LPS and CLP aortae [21, 29] however, the exact $\mathrm{Kv}$ isoform responsible for sepsis-induced vascular dysfunction has not been identified. We hypothesized that $\mathrm{Kv}_{1.5}$ could be a potential target for sepsis induced vascular hyporesponsiveness to PE since the $\mathrm{Kv}_{1.5}$ channel has been suggested as one of the major components of delayed rectifier $\mathrm{K}^{+}$currents in a variety of vascular beds $[33,34]$. Contrary to our hypothesis, inhibition of $\mathrm{Kv}_{1.5}$ by DPO-1 [35] was not able to improve PE hyporesponsiveness in $\mathrm{Cl}$ aorta. Similar to a previous study using CLP aorta [21], the nonselective $\mathrm{Kv}$ inhibitor, 4-AP, significantly increased PE responsiveness in $\mathrm{Cl}$ aorta (data not shown). These data suggest that $\mathrm{K}^{+}$channels other than the $\mathrm{Kv}_{1.5}$ channel may be responsible for $\mathrm{Cl}$-associated hyporesponsiveness to PE.

The ATP dependent $\mathrm{K}^{+}\left(\mathrm{K}_{\text {ATP }}\right)$ channel is another major $\mathrm{K}^{+}$channel in vascular smooth muscle cells that has been suggested to possibly undergo dysregulation in the rat CLP model [21]. Glibenclamide, a $\mathrm{K}_{\text {ATP }}$ channel inhibitor, significantly increased the PE response, suggesting that increased $\mathrm{K}_{\text {ATP }}$ channel activity, at least in part, contributed to Cl-induced hyporesponsiveness to PE ( $P<$ 0.05 vs Sham, Figure 5C).

\section{Sepsis associated changes in aortic structure}


Severe sepsis and endotoxemia both appeared to result in aortic damage and elevations in VCAM expression which is a marker for vascular endothelial inflammation (Figure 6B). Similar to the changes we observed in contractility, elevations in VCAM expression were similar in the Cl and CLP models which were both less than that observed with endotoxemia. Similarly, both sepsis and endotoxemia were associated with actin filament disorganization (Figure 7).

Our vascular reactivity demonstrated Ach response was different between polymicrobial and endotoxemia models, suggesting endothelial response may be different depending on the nature of the septic insult. In order to further investigate endothelial damage, VCAM expression, which is a marker for vascular endothelial inflammation, was determined. Although all septic models showed significant elevation of VCAM expression relative to each control group, the degree of increase was similar in the $\mathrm{Cl}$ and CLP models while the LPS model showed over a 7 -fold increase (Figure 6B). 


\section{Discussion}

The primary purpose of this study was to characterize vascular dysfunction produced in the $\mathrm{Cl}$ sepsis model. The polymicrobial $\mathrm{Cl}$ model was introduced 40 years ago as an alternative to the oftentimes variable CLP method of inducing sepsis [36]. Building upon the early cardiovascular characterization experiments done by Lang and colleagues [11], Sharma and co-workers have done a substantial amount of work to understand the mechanisms of cardiac dysfunction in the $\mathrm{Cl}$ model using pharmacological and genetic approaches [9, $10,37,38]$. Nonetheless, how vascular function may be affected in the $\mathrm{Cl}$ model is not well understood. The $\mathrm{Cl}$ experiment employs the use of a known amount of cecal material form a fresh donor anima, or preserved cecal material to allow a highly reproducible sepsis phenotype [8]. The advantages of this models Include a high degree of control over the severity of the sepsis insult as compared to CLP procedure and the use of a polymicrobial insult as opposed just a single agent as is seen in the LPS endotoxemia model.

One of the major findings of this study was that $\mathrm{Cl}$-induced sepsis is associated with vascular hyporesponsiveness to the $\alpha$-adrenergic agonist PE and that this impairment in vascular reactivity is characterized by a dysregulation of $\mathrm{K}^{+}$channel function. Similar to the commonly used LPS and CLP models, we found that L-NAME treatment increased PE response in the $\mathrm{Cl}$ model suggesting $\mathrm{NO}$ component in $\mathrm{Cl}$ vascular hypocontractility. Nonetheless, our data suggest that the decreased contractility seen in the $\mathrm{Cl}$ aorta does not appear to be solely dependent on NO or prostanoids, as incubation with L-NAME or inhibition of 
COX-2 was unable to restore tension production to a level similar to that seen in the non-septic animals.(Figures $2 A-C$ ).

In terms of endothelial function, the Ach response in the LPS and CLP models has been extensively studied [12, 39-44]. In this study, we found that Ach response was not affected in $\mathrm{Cl}$ and the CLP models. Studies have shown that Ach response was decreased in aorta at $5 \mathrm{~h}$ after CLP surgery $[43,44]$, while the Ach response was not different in aorta at $48 \mathrm{~h}$ after CLP [12]. Conversely, and consistent with previous reports [39-41], the LPS aorta showed significantly impaired Ach response (Figure 3C). Taken together, these data suggest that the mechanisms of sepsis-induced endothelial dysfunction seem to be different between CLP and LPS and that the mechanism(s) of Cl-induced vascular dysfunction may be similar to that observed in the CLP model. To further examine the effects of the $\mathrm{Cl}$ procedure on vascular endothelial dysfunction, we next examined VCAM expression. Similar to our aortic reactivity findings, we found that VCAM expression was significantly elevated in all three models and that the degree of elevation appeared to be different between the polymicrobial and endotoxemia models (Figure 6B).

To further characterize the function of smooth muscle in the $\mathrm{Cl}$ model, we next examined the contraction response in the denuded aorta. We found that contractile response to PE was significantly reduced in the absence of endothelial cells from $\mathrm{Cl}$ aorta (Figures $4 \mathrm{~A}, \mathrm{~B}$ ), suggesting that the $\mathrm{Cl}$ model is associated with impaired smooth muscle contraction. Interestingly, inhibition of NO caused a significantly increase in the contraction response generated by the 
$\mathrm{Cl}$ denuded aorta (Figures $4 \mathrm{~A}, \mathrm{~B}$ ). These data may suggest that the $\mathrm{Cl}$ model may be associated with not only the endothelial dysfunction, but also smooth muscle dysfunction. Indeed, this finding is similar to that noted by Chaudry and coworkers who demonstrated that smooth muscle contractile dysfunction during the later stages of sepsis was not restored in the absence of endothelial cell input [27]. In addition, recent research has also demonstrated that nNOS upregulation may play a role in CLP-induced vascular hyporesponsiveness [45]. Taken together, our observations from the $\mathrm{Cl}$ model are generally consistent with the notion that vascular hyporeactivity is seen in other commonly used preclinical septic models including CLP and LPS, however, each model may present specific mechanisms of vascular dysfunction.

Sepsis-induced alterations in $\mathrm{K}^{+}$channel function have been suggested as a potential mechanism to explain the vascular hyporesponsiveness seen with sepsis $[21,29,46,47]$. Whether the $\mathrm{Cl}$ model exhibits altered $\mathrm{K}^{+}$channel function has not been investigated. We found that aorta from the $\mathrm{Cl}$ animals exhibited an oscillating rhythmic contractions which was abolished following inhibition of the $\mathrm{BK}_{\mathrm{Ca}}$ channel (Figure 5B). Previous studies have reported similar oscillatory behavior from CLP aorta [32] and altered intracellular free calcium levels have been confirmed in this model [31]. We speculate that the Cl-induced rhythmic contraction could be due to altered $\mathrm{Ca}^{2+}$ levels as $\mathrm{BK}_{\mathrm{Ca}}$ channel inhibition can block the rhythmic contraction. We also found that inhibition of $\mathrm{Kv}_{1.5}$ channels did not normalize contractility, but that 4-AP significantly increased the tension developed after incubation with PE. These data suggest that other Kv families 
may play a role in the sepsis-associated hyporesponsiveness to PE. Finally, we noted that inhibition of the $\mathrm{K}_{\text {ATP }}$ channel could only partially restore sepsisinduced hyporesponsiveness to PE. Taken together, these data suggest that the $\mathrm{Cl}$ model is associated with hyperactivation of the $\mathrm{K}$ channels in the aorta.

In the present study, we found that pharmacological approaches could not completely restore vascular hypocontractility. Structural alterations in the vasculature have been shown in a number of different vascular disorders [48-50]. Both the organization of the actin cytoskeleton and the anatomical architecture of internal elastic lamina have been suggested to play a role in regulation of vascular function $[51,52]$. Indeed, cytochalasin has been shown to reduce rat aortic contraction, suggesting actin polymerization plays a role in vascular contraction [53]. We found that aorta from all sepsis models showed noticeable structural damage (Figures $6 A$ and 7 ). Although we cannot quantitatively explain to what degree these altered structures contributed to reduced aortic contractility in sepsis, sepsis-induced decreased aortic contractility may be due, at least in part, to these alterations in aortic structure. Further research is required to determine how sepsis induced structural alterations play a role in sepsis-induced hyporesponsiveness.

\section{Conclusion}

In conclusion, $\mathrm{Cl}$ procedure resulted in vascular phenotypic changes similar to that seen in the CLP procedure and LPS induced endotoxemia. However, this comparative study suggests that each model may present different 
mechanisms of vascular hyporeactivity, the degrees of vascular hyporeactivity and mortality rates. Thus, a single preclinical sepsis model may not be sufficient for the translational study. Although the exact mechanisms of sepsis vascular hyporeactivity remain unclear, the $\mathrm{Cl}$ model could serve as an alternative model for sepsis vascular dysfunction study.

\section{Conflict of interest statement}

The authors declare that there is no conflict of interests regarding the publication of this paper

\section{Acknowledgements}

Authors would like to acknowledge Steven Rogers, Niraj Nepal, and Mani Maheshwari for sample collections. This work was supported by DOE (DESC0005162) to ERB.

\section{References}

[1] D.C. Angus, W.T. Linde-Zwirble, J. Lidicker, G. Clermont, J. Carcillo, M.R. Pinsky, Epidemiology of severe sepsis in the United States: analysis of incidence, outcome, and associated costs of care, Critical care medicine, 29 (2001) 1303-1310.

[2] M. Varpula, M. Tallgren, K. Saukkonen, L.M. Voipio-Pulkki, V. Pettila, Hemodynamic variables related to outcome in septic shock, Intensive care medicine, 31 (2005) 1066-1071.

[3] A.B. Groeneveld, W. Bronsveld, L.G. Thijs, Hemodynamic determinants of mortality in human septic shock, Surgery, 99 (1986) 140-153.

[4] M.W. Dunser, J. Takala, H. Ulmer, V.D. Mayr, G. Luckner, S. Jochberger, F. Daudel, P. Lepper, W.R. Hasibeder, S.M. Jakob, Arterial blood pressure during early sepsis and outcome, Intensive care medicine, 35 (2009) 1225-1233.

[5] M.P. Fink, S.O. Heard, Laboratory models of sepsis and septic shock, The Journal of surgical research, 49 (1990) 186-196. 
[6] W.J. Hubbard, M. Choudhry, M.G. Schwacha, J.D. Kerby, L.W. Rue, 3rd, K.I. Bland, I.H. Chaudry, Cecal ligation and puncture, Shock, 24 Suppl 1 (2005) 52-57.

[7] L. Dejager, I. Pinheiro, E. Dejonckheere, C. Libert, Cecal ligation and puncture: the gold standard model for polymicrobial sepsis?, Trends in microbiology, 19 (2011) 198-208.

[8] M.E. Starr, A.M. Steele, M. Saito, B.J. Hacker, B.M. Evers, H. Saito, A new cecal slurry preparation protocol with improved long-term reproducibility for animal models of sepsis, PloS one, 9 (2014) e115705.

[9] A.C. Sharma, S.J. Motew, S. Farias, K.J. Alden, H.B. Bosmann, W.R. Law, J.L.

Ferguson, Sepsis alters myocardial and plasma concentrations of endothelin and nitric oxide in rats, Journal of molecular and cellular cardiology, 29 (1997) 14691477.

[10] M. Chopra, P. Das, A.C. Sharma, Caspase-3 knock-down reverses contractile dysfunction induced by sepsis in adult rat ventricular myocytes, British journal of pharmacology, 160 (2010) 93-100.

[11] C.H. Lang, G.J. Bagby, J.L. Ferguson, J.J. Spitzer, Cardiac output and redistribution of organ blood flow in hypermetabolic sepsis, The American journal of physiology, 246 (1984) R331-337.

[12] N. Nin, M. El-Assar, C. Sanchez, A. Ferruelo, A. Sanchez-Ferrer, L. Martinez-Caro, Y. Rojas, M. Paula, J. Hurtado, A. Esteban, J.A. Lorente, Vascular dysfunction in sepsis: effects of the peroxynitrite decomposition catalyst MnTMPyP, Shock, 36 (2011) 156161.

[13] D. Fernandes, R. Sordi, L.K. Pacheco, G.M. Nardi, B.T. Heckert, C.G. Villela, A.R. Lobo, C. Barja-Fidalgo, J. Assreuy, Late, but not early, inhibition of soluble guanylate cyclase decreases mortality in a rat sepsis model, The Journal of pharmacology and experimental therapeutics, 328 (2009) 991-999.

[14] R.M. Bateman, M.D. Sharpe, D. Goldman, D. Lidington, C.G. Ellis, Inhibiting nitric oxide overproduction during hypotensive sepsis increases local oxygen consumption in rat skeletal muscle, Critical care medicine, 36 (2008) 225-231. [15] T. Sigurdardottir, V. Bjorck, H. Herwald, M. Morgelin, S. Rutardottir, J. Tornebrant, M. Bodelsson, M1 protein from streptococcus pyogenes induces nitric oxide-mediated vascular hyporesponsiveness to phenylephrine: involvement of tolllike receptor activation, Shock, 34 (2010) 98-104.

[16] M.M. Lalu, J. Cena, R. Chowdhury, A. Lam, R. Schulz, Matrix metalloproteinases contribute to endotoxin and interleukin-1beta induced vascular dysfunction, British journal of pharmacology, 149 (2006) 31-42.

[17] J.J. Cena, M.M. Lalu, W.J. Cho, A.K. Chow, M.L. Bagdan, E.E. Daniel, M.M. Castro, R. Schulz, Inhibition of matrix metalloproteinase activity in vivo protects against vascular hyporeactivity in endotoxemia, American journal of physiology. Heart and circulatory physiology, 298 (2010) H45-51.

[18] T.M. McKenna, F.M. Martin, B. Chernow, F.A. Briglia, Vascular endothelium contributes to decreased aortic contractility in experimental sepsis, Circulatory shock, 19 (1986) 267-273.

[19] H. Taguchi, D.D. Heistad, Y. Chu, C.D. Rios, H. Ooboshi, F.M. Faraci, Vascular expression of inducible nitric oxide synthase is associated with activation of $\mathrm{Ca}(++)-$ 
dependent $\mathrm{K}+$ channels, The Journal of pharmacology and experimental therapeutics, 279 (1996) 1514-1519.

[20] W. Shi, N. Cui, Z. Wu, Y. Yang, S. Zhang, H. Gai, D. Zhu, C. Jiang, Lipopolysaccharides up-regulate Kir6.1/SUR2B channel expression and enhance vascular KATP channel activity via NF-kappaB-dependent signaling, The Journal of biological chemistry, 285 (2010) 3021-3029.

[21] J.H. Kuo, S.J. Chen, C.C. Shih, W.M. Lue, C.C. Wu, Abnormal activation of potassium channels in aortic smooth muscle of rats with peritonitis-induced septic shock, Shock, 32 (2009) 74-79.

[22] Guide for the Care and Use of Laboratory Animals, Washington (DC), 2011.

[23] R. Maeso, J. Navarro-Cid, R. Munoz-Garcia, E. Rodrigo, L.M. Ruilope, V. Lahera, V. Cachofeiro, Losartan reduces phenylephrine constrictor response in aortic rings from spontaneously hypertensive rats. Role of nitric oxide and angiotensin II type 2 receptors, Hypertension, 28 (1996) 967-972.

[24] E.R. Blough, K.M. Rice, D.H. Desai, P. Wehner, G.L. Wright, Aging alters mechanical and contractile properties of the Fisher 344/Nnia X Norway/Binia rat aorta, Biogerontology, 8 (2007) 303-313.

[25] S. Asano, J.D. Tune, G.M. Dick, Bisphenol A activates Maxi-K (K(Ca)1.1) channels in coronary smooth muscle, British journal of pharmacology, 160 (2010) 160-170. [26] S.R. Hyde, R.D. Stith, R.E. McCallum, Mortality and bacteriology of sepsis following cecal ligation and puncture in aged mice, Infection and immunity, 58 (1990) 619-624.

[27] S.H. Wurster, P. Wang, R.E. Dean, I.H. Chaudry, Vascular smooth muscle contractile function is impaired during early and late stages of sepsis, The Journal of surgical research, 56 (1994) 556-561.

[28] M.R. Farmer, R.E. Roberts, S.M. Gardiner, V. Ralevic, Effects of in vivo lipopolysaccharide infusion on vasoconstrictor function of rat isolated mesentery, kidney, and aorta, The Journal of pharmacology and experimental therapeutics, 306 (2003) 538-545.

[29] S.J. Chen, C.C. Wu, S.N. Yang, C.I. Lin, M.H. Yen, Hyperpolarization contributes to vascular hyporeactivity in rats with lipopolysaccharide-induced endotoxic shock, Life sciences, 68 (2000) 659-668.

[30] S. Hall, S. Turcato, L. Clapp, Abnormal activation of K+ channels underlies relaxation to bacterial lipopolysaccharide in rat aorta, Biochemical and biophysical research communications, 224 (1996) 184-190.

[31] S.K. Song, I.E. Karl, J.J. Ackerman, R.S. Hotchkiss, Increased intracellular Ca2+: a critical link in the pathophysiology of sepsis?, Proceedings of the National Academy of Sciences of the United States of America, 90 (1993) 3933-3937.

[32] B.J. Heesen, R.S. Hotchkiss, I.E. Karl, Sepsis decreases phenylephrine- and KClinduced aortic ring contraction and decreases the frequency of oscillations in active wall tension, Shock, 2 (1994) 106-112.

[33] G.M. Dick, I.N. Bratz, L. Borbouse, G.A. Payne, U.D. Dincer, J.D. Knudson, P.A. Rogers, J.D. Tune, Voltage-dependent K+ channels regulate the duration of reactive hyperemia in the canine coronary circulation, American journal of physiology. Heart and circulatory physiology, 294 (2008) H2371-2381. 
[34] T.T. Chen, K.D. Luykenaar, E.J. Walsh, M.P. Walsh, W.C. Cole, Key role of Kv1 channels in vasoregulation, Circulation research, 99 (2006) 53-60.

[35] G.L. Stump, A.A. Wallace, C.P. Regan, J.J. Lynch, Jr., In vivo antiarrhythmic and cardiac electrophysiologic effects of a novel diphenylphosphine oxide IKur blocker (2-isopropyl-5-methylcyclohexyl) diphenylphosphine oxide, The Journal of pharmacology and experimental therapeutics, 315 (2005) 1362-1367.

[36] A.B. Onderdonk, W.M. Weinstein, N.M. Sullivan, J.G. Bartlett, S.L. Gorbach, Experimental intra-abdominal abscesses in rats: quantitative bacteriology of infected animals, Infection and immunity, 10 (1974) 1256-1259.

[37] M. Chopra, H.B. Golden, S. Mullapudi, W. Dowhan, D.E. Dostal, A.C. Sharma, Modulation of myocardial mitochondrial mechanisms during severe polymicrobial sepsis in the rat, PloS one, 6 (2011) e21285.

[38] A. Gupta, N.S. Aberle, 2nd, J. Ren, A.C. Sharma, Endothelin-converting enzyme-1mediated signaling in adult rat ventricular myocyte contractility and apoptosis during sepsis, Journal of molecular and cellular cardiology, 38 (2005) 527-537. [39] J.L. Parker, H.R. Adams, Selective inhibition of endothelium-dependent vasodilator capacity by Escherichia coli endotoxemia, Circulation research, 72 (1993) 539-551.

[40] G. Julou-Schaeffer, G.A. Gray, I. Fleming, C. Schott, J.R. Parratt, J.C. Stoclet, Loss of vascular responsiveness induced by endotoxin involves L-arginine pathway, The American journal of physiology, 259 (1990) H1038-1043.

[41] P.R. Myers, Q. Zhong, J.J. Jones, M.A. Tanner, H.R. Adams, J.L. Parker, Release of EDRF and NO in ex vivo perfused aorta: inhibition by in vivo E. coli endotoxemia, The American journal of physiology, 268 (1995) H955-961.

[42] S.J. Chen, C.C. Wu, M.H. Yen, Alterations of ex vivo vascular reactivity in intraperitoneal sepsis, Journal of cardiovascular pharmacology, 24 (1994) 786-793. [43] A.M. Morrison, P. Wang, I.H. Chaudry, A novel nonanticoagulant heparin prevents vascular endothelial cell dysfunction during hyperdynamic sepsis, Shock, 6 (1996) 46-51.

[44] P. Wang, Z.F. Ba, I.H. Chaudry, Endothelium-dependent relaxation is depressed at the macro- and microcirculatory levels during sepsis, The American journal of physiology, 269 (1995) R988-994.

[45] G.M. Nardi, K. Scheschowitsch, D. Ammar, S.K. de Oliveira, T.B. Arruda, J. Assreuy, Neuronal nitric oxide synthase and its interaction with soluble guanylate cyclase is a key factor for the vascular dysfunction of experimental sepsis, Critical care medicine, 42 (2014) e391-400.

[46] C.C. Wu, C. Thiemermann, J.R. Vane, Glibenclamide-induced inhibition of the expression of inducible nitric oxide synthase in cultured macrophages and in the anaesthetized rat, British journal of pharmacology, 114 (1995) 1273-1281. [47] C.C. Wu, S.J. Chen, M.H. Yen, Nitric oxide-independent activation of soluble guanylyl cyclase contributes to endotoxin shock in rats, The American journal of physiology, 275 (1998) H1148-1157.

[48] R. Christie, M. Yamada, M. Moskowitz, B. Hyman, Structural and functional disruption of vascular smooth muscle cells in a transgenic mouse model of amyloid angiopathy, The American journal of pathology, 158 (2001) 1065-1071. 
[49] P. Lacolley, V. Regnault, A. Nicoletti, Z. Li, J.B. Michel, The vascular smooth muscle cell in arterial pathology: a cell that can take on multiple roles, Cardiovascular research, 95 (2012) 194-204.

[50] G.F. Mitchell, Effects of central arterial aging on the structure and function of the peripheral vasculature: implications for end-organ damage, Journal of applied physiology, 105 (2008) 1652-1660.

[51] B.S. Kirby, A. Bruhl, M.N. Sullivan, M. Francis, F.A. Dinenno, S. Earley, Robust internal elastic lamina fenestration in skeletal muscle arteries, PloS one, 8 (2013) e54849.

[52] V. Sauzeau, H. Le Jeune, C. Cario-Toumaniantz, A. Smolenski, S.M. Lohmann, J. Bertoglio, P. Chardin, P. Pacaud, G. Loirand, Cyclic GMP-dependent protein kinase signaling pathway inhibits RhoA-induced $\mathrm{Ca} 2+$ sensitization of contraction in vascular smooth muscle, The Journal of biological chemistry, 275 (2000) 2172221729.

[53] G. Wright, E. Hurn, Cytochalasin inhibition of slow tension increase in rat aortic rings, The American journal of physiology, 267 (1994) H1437-1446. 